\title{
MULTIPLE POSITIVE SOLUTIONS FOR A CLASS OF VARIATIONAL SYSTEMS
}

\author{
Alfonso Castro - David Costa - Ratnasingham Shivaji
}

\begin{abstract}
We consider the variational system $-\Delta u=\lambda(\nabla F)(u)$ in $\Omega$, $u=0$ on $\partial \Omega$, where $\Omega$ is a bounded region in $\mathbb{R}^{m}(m \geq 1)$ with $C^{1}$ boundary, $\lambda$ is a positive parameter, $u: \Omega \rightarrow \mathbb{R}^{N}(N>1)$, and $\Delta$ denotes the Laplace operator. Here $F: \mathbb{R}^{N} \rightarrow \mathbb{R}$ is a function of class $C^{2}$. Using variational methods, we show how changes in the sign of $F$ lead to multiple positive solutions.
\end{abstract}

\section{Introduction}

We study the existence of positive solutions to the variational system

$$
\begin{cases}-\Delta u=\lambda(\nabla F)(u) & \text { in } \Omega, \\ u=0 & \text { on } \partial \Omega,\end{cases}
$$

where $\Omega$ is a bounded region in $\mathbb{R}^{m}(m \geq 1)$ with $C^{1}$ boundary, $\lambda$ is a positive parameter, $u: \Omega \rightarrow \mathbb{R}^{N}(N>1)$, and $\Delta$ denotes the Laplacian operator. Here $F: \mathbb{R}^{N} \rightarrow \mathbb{R}$ is a function of class $C^{2}$. By a positive solution to (1.1) we mean a function $u=\left(u_{1}, \ldots, u_{N}\right)$ with each $u_{j} \in C^{2}(\Omega) \cap C^{1}(\bar{\Omega}), u_{j}(x) \geq 0 ; \Omega$, $u_{j}(x)=0 ; \partial \Omega$ and $u_{l}\left(x_{0}\right)>0$ for some $l \in\{1, \ldots, N\}, x_{0} \in \Omega$.

2010 Mathematics Subject Classification. 35J20, 35J50.

Key words and phrases. Positive solutions; systems; variational method.

The first named author was partially supported by a grant from the Simons Foundation (\# 245966 to Alfonso Castro).

The third named author was partially supported by a grant from the Simons Foundation (\# 317872 to Ratnasingham Shivaji). 
Let $\Sigma=\left\{z=\left(z_{1}, \ldots, z_{N}\right) \mid z_{1}+\ldots+z_{N}=1\right.$ with $z_{i} \geq 0$ for all $\left.i=1, \ldots, N\right\}$ and $Q=\left\{z=\left(z_{1}, \ldots, z_{N}\right) \mid z_{i}>0\right.$ for all $\left.i=1, \ldots, N\right\}$. For $z \in \mathbb{R}^{N}$ we will denote $|z|_{1}=\left|z_{1}\right|+\ldots+\left|z_{N}\right|$ and $P(z)=z /|z|_{1}$, for $z \neq 0$. We assume:

(H1) $F\left(z_{1}, \ldots, z_{N}\right)=0$ if $z_{1} \ldots z_{N}=0$, and $F\left(z_{1}, \ldots, z_{N}\right) \leq 0$ if $z_{i} \leq 0$ for some $i=1, \ldots, N$.

(H2) There exist concave functions $\rho_{1}, \ldots, \rho_{2 k}: \Sigma \rightarrow[0, \infty)$ such that

$$
0<\rho_{i}(z)<\rho_{i+1}(z), \quad i=1, \ldots, 2 k-1 .
$$

(H3) For $z \in Q, F(z)>0$ if $|z|_{1} \in\left(\rho_{2 i+1}(P(z)), \rho_{2(i+1)}(P(z))\right)$, and $F(z)<$ 0 if $|z|_{1} \in\left(\rho_{2 i}(P(z)), \rho_{2 i+1}(P(z))\right)$ for $i=0,2, \ldots, k-1$ or $|z|_{1} \geq$ $\rho_{2 k}(P(z))$.

(H4) For $i=0, \ldots, k-1$ there exists $z^{(i)} \in Q$ such that $\left|z^{(i)}\right|_{1} \in\left(\rho_{2 i+1}\left(P\left(z^{(i)}\right)\right)\right.$, $\left.\rho_{2(i+1)}\left(P\left(z^{(i)}\right)\right)\right)$ and $F\left(z^{(i)}\right)>\max \left\{\left.F(z)|| z\right|_{1}<\rho_{2 i+1}\left(P\left(z^{(i)}\right)\right)\right\}$.

Our main result is the following:

THeOREM 1.1. There exist $\lambda_{1}<\ldots<\lambda_{k}$ such that if $\lambda>\lambda_{i}$, for $i=$ $1, \ldots, k-1$, then the boundary value problem (1.1) has $i$ positive solutions.

In the single equation case $(N=1)$ there is a rich history on the study of such boundary value problems where the analysis of how the changes of sign of the nonlinear term give rise to the existence of multiple positive solutions. In particular see Brown-Budin [1] where a combination of variational and monotone iteration methods is used, Hess [8] where a combination of variational and topological degree arguments is applied, and De Figueiredo [7] where only variational methods are used. Clement and Sweers in [3] proved that if $f:[0, \infty) \rightarrow \mathbb{R}$ satisfies

$$
\begin{array}{ll}
f(s)<0 & \text { for } 0<s<s_{1} \text { or } s>s_{2}, \\
f(s)>0 & \text { for } s_{1}<s<s_{2},
\end{array}
$$

for some $0<s_{1}<s_{2}$, then the (possibly semipositone) problem

$$
-\Delta u=\lambda f(u) \quad \text { in } \Omega, \quad u(x)=0 \quad \text { for } x \in \partial \Omega,
$$

has a positive solution $u$ for $\lambda$ large with $\|u\|_{\infty} \in\left(s_{1}, s_{2}\right)$ if and only if

$$
\int_{0}^{s_{2}} f(s) d s>0 .
$$

Also, Dancer and Schmitt in [5] used sub-supersolutions to show that (1.4) is necessary for existence of a positive solution to (1.3). In [6], De Figueiredo showed that for $f(u)=\sin (u)$ and $\lambda>0$ in (1.3) this equation has only one positive solution. However, to date no extension of this study has been achieved for the system case (when $N>1$ ), which we establish in this paper via variational methods. 
For related results on $2 \times 2$ systems the reader is referred to [2] and [4]. In [2], extensive use of regularity properties of elliptic operators and the Krasnosel'skiı compression-expansion theorem is made to prove the existence of positive solutions. In [4], variational methods are employed in proving existence of solutions for non-cooperative systems.

In Section 2 we establish auxiliary lemmas needed for the proof of Theorem 1.1 in Section 3.

\section{Auxiliary results}

For $j=1, \ldots, k$ let $S_{j}$ be the closure of $\left\{\left.z \in Q|| z\right|_{1} \leq \rho_{2 j}(P(z))\right\}$. Also, let $S_{0}$ be the closure of $\left\{\left.z \in Q|| z\right|_{1} \leq \rho_{1}(P(z))\right\}$. From (H2) it follows that $S_{j}$ is convex. We will denote by $P_{j}$ the projection of $\mathbb{R}^{N}$ onto the convex set $S_{j}$. Hence $P_{j}$ is locally Lipschitzian. Let

$$
F_{j}(u):= \begin{cases}0 & \text { if } u \notin S_{j}, \\ F(u) & \text { if } u \in S_{j} .\end{cases}
$$

Since $F$ is differentiable, $F_{j}$ is locally Lipschitzian and the functional

$$
J_{j, \lambda}: H=\left(H_{0}^{1,2}(\Omega)\right)^{N} \rightarrow \mathbb{R}
$$

defined by

$$
J_{j, \lambda}(u)=\int_{\Omega}\left(\frac{1}{2} \sum_{i=1}^{N}\left|\nabla u_{i}\right|^{2}-\lambda F_{j}(u)\right) d x
$$

attains its minimum value. Indeed, $J_{j, \lambda}$ is bounded below because $F_{j}$ is bounded. If $\left\{J_{j, \lambda}\left(v_{k}\right)\right\}$ converges to $\inf \left\{J_{j, \lambda}(u) \mid u \in H\right\}$ then $\left\{v_{k}\right\}$ is bounded in $H$. Hence it has a subsequence (call it $\left\{v_{k}\right\}$ again) that converges in $L^{2}(\Omega)$ to some $\widehat{u}_{j}$. Hence $\left\{\int_{\Omega} F_{j}\left(v_{k}\right) d x\right\}$ converges to $\int_{\Omega} F_{j}\left(\widehat{u}_{j}\right) d x$. This and the convexity of the norm gives $J_{j, \lambda}\left(\widehat{u}_{j}\right)=\min \left\{J_{j, \lambda}(u) \mid u \in H\right\}$.

Lemma 2.1. If $\widehat{u}_{j}:=\left(\widehat{u}_{j, 1}, \ldots, \widehat{u}_{j, N}\right)$ is as above, then $\widehat{u}_{j}(\zeta) \in \bar{S}_{j}$ for all $\zeta \in \Omega$.

Proof. For the sake of simplicity in the notation, throughout this proof we denote $\widehat{u}_{j}=u, \widehat{u}_{j, l}=u_{l}$, and $J_{j, \lambda}=J$. Suppose first that $v=\left(u_{l}\right)_{-}$is not zero for some $l \in\{1, \ldots, N\}$. Hence, there exists $c>0$ such that

$$
\int_{\Omega_{c}}|\nabla v|^{2} d x<\int_{\Omega}|\nabla v|^{2} d x
$$

where $\Omega_{c}=\{x \in \Omega \mid v(x) \leq c\}$. Let $w=\min \{v, c\}$. Thus

$$
w=\left(u_{1}, \ldots, u_{l-1}, v, u_{l+1}, \ldots, u_{N}\right) \in H .
$$


By the definition of $F_{j}$ we have $F_{j}(u(x))=F_{j}(w(x))$ for all $x \in \Omega$. Therefore,

$$
\begin{aligned}
J(w) & =\frac{1}{2} \int_{\Omega} \sum_{k=1, k \neq l}^{N}\left|\nabla u_{k}\right|^{2} d x+\frac{1}{2} \int_{\Omega}|\nabla v|^{2} d x-\lambda \int_{\Omega} F_{j}(w) d x \\
& <\frac{1}{2} \int_{\Omega} \sum_{k=1}^{N}\left|\nabla u_{k}\right|^{2} d x-\lambda \int_{\Omega} F_{j}(u) d x=J(u),
\end{aligned}
$$

which contradicts the definition of $u$. Hence $u_{l} \geq 0$ for all $l=1, \ldots, N$.

Suppose next that the Lebesgue measure of $W=\left\{x \in \Omega \mid u(x) \notin S_{j}\right\}$ is positive. Since $S_{j}$ is convex there exist $\eta_{1} \in \mathbb{R}^{N}, \mu \in(0,+\infty)$, such that the Lebesgue measure of $W_{1}=\left\{x \in \Omega \mid u(x) \cdot \eta_{1}>\mu\right\}$ is positive. Hence

$$
\int_{W_{1}} \sum_{l=1, i=1}^{N, N}\left(\frac{\partial u_{l}}{\partial x_{i}}(x)\right)^{2} d x>0 .
$$

Let $w(x)=u(x)-\left(\left(u(x) \cdot \eta_{1}\right)-\mu\right) \eta_{1}$ for $\left(u(x) \cdot \eta_{1}\right) \geq \mu$ and $w(x)=u(x)$ for $\left(u(x) \eta_{1}\right) \leq \mu$. Thus $w \in H$. Since $W_{1}$ is a subset of the complement of $S_{j}$, $F_{j}(w(x))=F_{j}(u(x))$ for all $x \in \Omega$. Let $\eta_{2}, \ldots, \eta_{N}$ be such that $\left\{\eta_{1}, \eta_{2}, \ldots, \eta_{N}\right\}$ is a complete orthonormal set in $\mathbb{R}^{N}$. Since

$$
u(x)=\left(u(x) \cdot \eta_{1}\right) \eta_{1}+\ldots+\left(u(x) \cdot \eta_{N}\right) \eta_{N},
$$

we have for $i=1, \ldots, N$ that

$$
\frac{\partial u}{\partial x_{i}}(x)=\left(\frac{\partial u}{\partial x_{i}}(x) \cdot \eta_{1}\right) \eta_{1}+\ldots+\left(\frac{\partial u}{\partial x_{i}}(x) \cdot \eta_{N}\right) \eta_{N} .
$$

Also, from the definition of $w$, for $x \in W_{1}$ and $i=1, \ldots, N$ we have

$$
\frac{\partial w}{\partial x_{i}}(x)=\left(\frac{\partial u}{\partial x_{i}}(x) \cdot \eta_{2}\right) \eta_{2}+\ldots+\left(\frac{\partial u}{\partial x_{i}}(x) \cdot \eta_{N}\right) \eta_{N} .
$$

Hence, letting $w_{1}, \ldots, w_{N}$ denote the components of $w$,

$$
\sum_{l=1, k=1}^{N, N}\left(\frac{\partial w_{l}}{\partial x_{i}}(x)\right)^{2} d x=\sum_{l=1, k=2}^{N, N}\left(\frac{\partial u}{\partial x_{i}} \cdot \eta_{k}\right)^{2},
$$

for al $x \in W_{1}$. Since $W_{1}$ is a set of positive measure,

$$
\int_{W_{1}} \sum_{i=1}^{N}\left(\frac{\partial \widehat{u}_{j}}{\partial x_{i}} \cdot \eta_{1}\right)^{2} d x>0 .
$$


Thus

$$
\begin{aligned}
\int_{W_{1}} \sum_{i=1, k=1}^{N, N} & \left(\frac{\partial u_{k}}{\partial x_{i}}\right)^{2} d x=\int_{W_{1}} \sum_{i=1}^{N}\left|\frac{\partial u}{\partial x_{i}}\right|^{2} d x \\
= & \int_{W_{1}} \sum_{i=1, k=1}^{N, N}\left(\frac{\partial u}{\partial x_{i}} \cdot \eta_{k}\right)^{2} d x>\int_{W_{1}} \sum_{i=1, k=2}^{N, N}\left(\frac{\partial u}{\partial x_{i}} \cdot \eta_{k}\right)^{2} d x \\
= & \int_{W_{1}} \sum_{i=1}^{N}\left|\frac{\partial w}{\partial x_{i}}\right|^{2} d x=\int_{W_{1}} \sum_{i=1, k=1}^{N, N}\left(\frac{\partial w_{k}}{\partial x_{i}}\right)^{2} d x
\end{aligned}
$$

Also, from the definition of $w$,

$$
\int_{W_{1}}\left(\nabla w_{i} \cdot \eta_{1}\right)^{2} d x=0
$$

for all $i=1, \ldots, N$. Letting $W_{2}=\Omega-W_{1}$, we have by (2.4) that

$$
\begin{aligned}
J(w) & =\int_{W_{2}}\left(\frac{1}{2} \sum_{k=1}^{N}\left|\nabla w_{k}\right|^{2}-\lambda F_{j}(w)\right) d x+\int_{W_{1}}\left(\frac{1}{2} \sum_{k=1}^{N}\left|\nabla w_{k}\right|^{2}\right) d x \\
& =\int_{W_{2}}\left(\frac{1}{2} \sum_{k=1}^{N}\left|\nabla u_{k}\right|^{2}-\lambda F_{j}\left(\widehat{u}_{j}\right)\right) d x+\frac{1}{2} \int_{W_{1}} \sum_{i, k=1}^{N}\left(\frac{\partial u_{i}}{\partial x_{k}}\right)^{2} d x \\
& <\frac{1}{2} \int_{\Omega} \sum_{k=1}^{N}\left|\nabla u_{k}\right|^{2} d x-\lambda \int_{\Omega} F_{j}\left(\widehat{u}_{j}\right) d x=J\left(\widehat{u}_{j}\right),
\end{aligned}
$$

Since this contradicts the definition of $u,(2.3)$ is impossible. Therefore the Lebesgue measure of $W$ is zero, which proves the lemma.

LEMmA 2.2. If $\widehat{u}_{j}:=\left(\widehat{u}_{j, 1}, \ldots, \widehat{u}_{j, N}\right)$ is as above, then $\widehat{u}_{j}$ is a solution to system (1.1).

Proof. Let $\phi: \Omega \rightarrow \mathbb{R}^{N}$ be a function of compact support and class $C^{\infty}$. By (H1) and Lemma 2.1, for $|t|$ sufficiently small we have

$$
F_{j}\left(\left(\widehat{u}_{j}+t \phi\right)(\zeta)\right) \geq F\left(\left(\widehat{u}_{j}+t \phi\right)(\zeta)\right)
$$

for all $\zeta \in \Omega$. Therefore,

$$
\begin{aligned}
I_{\phi}(t) & =J_{\lambda}\left(\widehat{u}_{j}+t \phi\right)=\frac{1}{2} \int_{\Omega}\left|\nabla\left(\widehat{u}_{j}+t \phi\right)\right|^{2} d \zeta-\lambda \int_{\Omega} F\left(\widehat{u}_{j}+t \phi\right) d \zeta \\
& \geq \frac{1}{2} \int_{\Omega}\left|\nabla\left(\widehat{u}_{j}+t \phi\right)\right|^{2} d \zeta-\lambda \int_{\Omega} F_{j}\left(\widehat{u}_{j}+t \phi\right) d \zeta=J_{j, \lambda}\left(\widehat{u}_{j}\right)=J_{\lambda}\left(\widehat{u}_{j}\right)=I_{\phi}(0),
\end{aligned}
$$

where

$$
J_{\lambda}(v)=\frac{1}{2} \int_{\Omega}|\nabla(v)|^{2} d \zeta-\lambda \int_{\Omega} F(v) d \zeta
$$

Therefore $I_{\phi}$ has a local minimum at $t=0$. Thus $0=I_{\phi}^{\prime}(0)=\left\langle\nabla J_{\lambda}\left(\widehat{u}_{j}\right), \phi\right\rangle$, which proves that $\widehat{u}_{j}$ is a weak solution to (1.1). Since $\nabla F$ is bounded in $\bar{S}_{j}$, by elliptic regularity for second order equations, $\widehat{u}_{j}$ is actually a solution to (1.1). 


\section{Proof of Theorem 1.1}

Proof. By Lemma 2.1, it is sufficient to show that

(A) There exists $\lambda_{i}, i=1, \ldots, k$, such that $\lambda_{1}<\ldots<\lambda_{i}$ and $J_{i, \lambda}\left(\widehat{u}_{i}\right)<$ $\ldots<J_{2, \lambda}\left(\widehat{u}_{2}\right)<J_{1, \lambda}\left(\widehat{u}_{1}\right)<0$ for $\lambda>\lambda_{i}$.

Let $N(x)$ denote the inward unit normal to $\partial \Omega$ at $x \in \partial \Omega$. Since $\partial \Omega$ is $C^{1}$ there exists $\varepsilon>0$ such that $\{x+s N(x) \mid s \in[0, \varepsilon)\}$ is an open neighborhood of $\partial \Omega$ relatively to $\bar{\Omega}$ and $x+s N(x) \neq y+t N(y)$ for $(x, s) \neq(y, t), x, y \in \partial \Omega$, $s, t \in[0, \varepsilon)$. Letting $\Omega_{\delta}=\{x \in \Omega \mid \operatorname{dist}(x, \partial \Omega)<\delta\}$ with $\delta \in(0, \varepsilon)$, we see that there exists a constant $M>0$ such that

$$
\left|\Omega_{\delta}\right| \leq M \delta
$$

Let $\widehat{u}_{j}$ be as in Lemma 2.1. Let us first see that there exists $\lambda_{1}>0$ such that $J_{1, \lambda}\left(\widehat{u}_{1}\right)<0$ for $\lambda>\lambda_{1}$. Using (H4) there exists $z^{(1)} \in S_{1}$ such that $F\left(z^{(1)}\right)>\max \left\{F(u) \mid u \in S_{0}\right\}$. Let $c=F\left(z^{(1)}\right)-\max \left\{F(u) \mid u \in S_{0}\right\}$. Now fix $\delta \in\left(0, \min \left\{\varepsilon, c|\Omega| /\left(2 M\left[c+M_{1}\right]\right)\right\}\right)$ where $M_{1}=\max _{u \in S_{1}}|F(u)|$ and let $v$ be defined by:

$$
v(x)= \begin{cases}z^{(1)} & \text { if } \operatorname{dist}(x, \partial \Omega) \geq \delta \\ \frac{s}{\delta} z^{(1)} & \text { if } \operatorname{dist}(x, \partial \Omega)<\delta, x=y+s N(y), y \in \partial \Omega, s \in[0, \delta) .\end{cases}
$$

Since $\partial \Omega$ is $C^{1}, v$ is a Lipschitzian function and there exists $K>0$ such that

$$
\int_{\Omega} \frac{1}{2} \sum_{i=1}^{N}\left|\nabla v_{i}\right|^{2} d x \leq K \delta^{-1}
$$

Then

$$
\begin{aligned}
J_{1, \lambda}(v) & =\int_{\Omega}\left(\frac{1}{2} \sum_{i=1}^{N}\left|\nabla v_{i}\right|^{2}-\lambda F_{1}(v)\right) d x \\
& \leq K \delta^{-1}-\lambda\left(\int_{\Omega-\Omega_{\delta}} F_{1}\left(z^{(1)}\right) d x+\int_{\Omega_{\delta}} F_{1}(v) d x\right) \\
& \leq K \delta^{-1}-\lambda\left[c\left(|\Omega|-\left|\Omega_{\delta}\right|\right)-\left|\Omega_{\delta}\right| M_{1}\right] \\
& \leq K \delta^{-1}-\lambda\left[c|\Omega|-c M \delta-M \delta M_{1}\right] \leq K \delta^{-1}-\lambda \frac{c|\Omega|}{2}
\end{aligned}
$$

since $\delta<c|\Omega| /\left(2 M\left[c+M_{1}\right]\right)<0$ for $\lambda>\lambda_{1}=2 K /(c|\Omega| \delta)$. Hence $J_{1, \lambda}\left(\widehat{u}_{1}\right)<0$ for $\lambda>\lambda_{1}$.

Next we show that there exists $\widetilde{\lambda}_{2}>\lambda_{1}$ such that $J_{1, \lambda}\left(\widehat{u}_{1}\right)>J_{2, \lambda}\left(\widehat{u}_{2}\right)$ for $\lambda>\widetilde{\lambda}_{2}$. Using (H4) there exists $z^{(2)} \in S_{2}$ such that $F\left(z^{(2)}\right)>\max \{F(u) \mid$ $\left.u \in S_{1}\right\}$. 
Now let $c=F\left(z^{(2)}\right)-\max \left\{F(u) \mid u \in S_{1}\right\} . \quad F i x \quad \delta \in(0, \min \{\varepsilon,(c|\Omega| /$ $\left.\left.\left(2 M\left[c+M_{1}+M_{2}\right]\right)\right\}\right)$ where $M_{2}=\max _{u \in S_{1}}|F(u)|$ and let

(3.4) $v(x)= \begin{cases}z^{(2)} & \text { if } \operatorname{dist}(x, \partial \Omega) \geq \delta, \\ \frac{s}{\delta} z^{(2)} & \text { if } \operatorname{dist}(x, \partial \Omega)<\delta, x=y+s N(y), y \in \partial \Omega, s \in[0, \delta) .\end{cases}$

Again, using that $\partial \Omega$ is $C^{1}$, we have a constant $K>0$ such that

$$
\int_{\Omega} \frac{1}{2} \sum_{i=1}^{N}\left|\nabla v_{i}\right|^{2} d x \leq K \delta^{-1}
$$

Then

$$
\begin{aligned}
J_{2, \lambda}(v) & =\int_{\Omega}\left(\frac{1}{2} \sum_{i=1}^{N}\left|\nabla v_{i}\right|^{2}-\lambda F_{2}(v)\right) d x \\
& \leq K \delta^{-1}-\lambda\left(\int_{\Omega-\Omega_{\delta}} F_{2}\left(z^{(2)}\right) d x+\int_{\Omega_{\delta}} F_{2}(v) d x\right) \\
& \leq K \delta^{-1}-\lambda\left(\int_{\Omega-\Omega_{\delta}}\left[F_{1}\left(\widehat{u}_{1}\right)+c\right] d x+\int_{\Omega_{\delta}} F_{2}(v) d x\right) \\
& =K \delta^{-1}-\lambda\left(\int_{\Omega} F_{1}\left(\widehat{u}_{1}\right) d x+c\left[|\Omega|-\left|\Omega_{\delta}\right|\right]+\int_{\Omega_{\delta}}\left[F_{2}(v)-F_{1}\left(\widehat{u}_{1}\right)\right] d x\right) \\
& \left.<K \delta^{-1}+J_{1, \lambda}\left(\widehat{u}_{1}\right)-\lambda\left\{c|| \Omega|-| \Omega_{\delta} \mid\right]-\left|\Omega_{\delta}\right|\left[M_{1}+M_{2}\right]\right\} \\
& <K \delta^{-1}+J_{1, \lambda}\left(\widehat{u}_{1}\right)-\lambda\left\{c|\Omega|-c M \delta-M \delta\left[M_{1}+M_{2}\right]\right\} \\
& <K \delta^{-1}+J_{1, \lambda}\left(\widehat{u}_{1}\right)-\lambda \frac{c|\Omega|}{2} \quad \text { since } \delta<\frac{c|\Omega|}{2 M\left[c+M_{1}+M_{2}\right]} \\
& <J_{1, \lambda}\left(\widehat{u}_{1}\right) \quad \text { for } \lambda>\widetilde{\lambda}_{2}=\frac{2 K}{c|\Omega| \delta} .
\end{aligned}
$$

Hence $J_{2, \lambda}\left(\widehat{u}_{2}\right)<J_{1, \lambda}\left(\widehat{u}_{1}\right)$ for $\lambda>\widetilde{\lambda}_{2}$. Now choosing $\lambda_{2}=\max \left\{\lambda_{1}, \widetilde{\lambda}_{2}\right\}$, for $\lambda>\lambda_{2}$ we have $J_{2, \lambda}\left(\widehat{u}_{2}\right)<J_{1, \lambda}\left(\widehat{u}_{1}\right)<0$. Iterating this argument $k$ times, (A) is proven and so is Theorem 1.1.

Finally, in order to state a consequence of Theorem 1.1, we consider the following extensions of $(\mathrm{H} 2)-(\mathrm{H} 4)$ :

$\widehat{(\mathrm{H} 2)}$ There exist concave functions $\rho_{i}: \Sigma \rightarrow[0, \infty)$ such that $0<\rho_{i}(z)<$ $\rho_{i+1}(z)$ for $i \in \mathbb{N}$.

$\widehat{\text { (H3) }}$ For $z \in Q$, assume $F(z)>0$ if $|z|_{1} \in\left(\rho_{2 i+1}(P(z)), \rho_{2(i+1)} l(P(z))\right)$, and $F(z)<0$ if $|z|_{1} \in\left(\rho_{2 i}(P(z)), \rho_{2 i+1}(P(z))\right)$ for each $i=0,1, \ldots$

$\widehat{(\mathrm{H} 4)}$ For each $i=0,1, \ldots$ there exists $z^{(i)} \in Q$ such that

$$
\left|z^{(i)}\right|_{1} \in\left(\rho_{2 i+1}\left(P\left(z^{(2(i+1))}\right)\right), \rho_{2(i+1)}\left(P\left(z^{(i)}\right)\right)\right)
$$

and $F\left(z^{(i)}\right)>\max \left\{\left.F(z)|| z\right|_{1}<\rho_{2 i+1}\left(P\left(z^{(i)}\right)\right)\right\}$.

The following result is an immediate corollary of Theorem 1.1: 
Theorem 3.1. Assume $(\mathrm{H} 1)$ and $\widehat{(\mathrm{H} 2)}-\widehat{(\mathrm{H} 4)}$. If we let $N(\lambda)$ denote the number of positive solutions of (1.1) then $N(\lambda) \rightarrow \infty$ as $\lambda \rightarrow \infty$.

\section{REFERENCES}

[1] K.J. Brown And H. Budin, On the existence of positive solutions for a class of semilinear elliptic boundary value problems, SIAM J. Math. Anal. 10 (1979), no. 5, 875-883.

[2] J.C. CARdEÑO AND A. CASTRO, Existence of positive solutions for a superlinear elliptic system with Neumann boundary condition, (English summary) Proceedings of the Variational and Topological Methods: Theory, Applications, Numerical Simulations, and Open Problems, 23-30, Electron. J. Differential Equations Conf. 21, Texas State University, San Marcos, TX, 2014.

[3] P. Clément And G. Sweers, Existence and multiplicity results for a semilinear elliptic eigenvalue problem, Ann. Scuola Norm. Sup. Pisa Cl. Sci. (4) 14 (1987), no. 1, 97-121.

[4] D. Costa and C. Magalhaes, A variational approach to noncooperative elliptic systems, Nonlinear Anal. 25 (1995), no. 7, 699-715.

[5] E.N. Dancer And K. Schmitt, On positive solutions of semilinear elliptic equations, Proc. Amer. Math. Soc. 101 (1987), no. 3, 445-452.

[6] D.G. De Figueiredo, On the Uniqueness of Positive Solutions of the Dirichlet Problem for $-\Delta u=\lambda \sin u$, Nonlinear Partial Differential Equations and their Applications, College de France Seminar, Vol. VII (Paris, 1983-1984), 80-83; Res. Notes in Math. 122, Pittman, 1985.

[7] _ On the existence of multiple ordered solutions of nonlinear eigenvalue problems, Nonlinear Anal. 11, (1987), no. 4, 481-492.

[8] P. Hess, On multiple positive solutions of nonlinear elliptic eigenvalue problems, Partial Differential Equations 6 (1981), 951-961.

[9] P.H. Rabinowitz, Minimax methods in Critical Point Theory with Applications to Differential Equations, Regional Conference Series in Mathematics, vol. 65, AMS, Providence (1986).

Alfonso Castro

Department of Mathematics

Harvey Mudd College

Claremont, CA 91711, USA

E-mail address: castro@math.hmc.edu

DAvid Costa

Department of Mathematics

University of Nevada Las Vegas

Las Vegas, NV 89154, USA

E-mail address: costa@unlv.nevada.edu

RAtNASINGHAM SHIVAJI

Department of Mathematics and Statistics University of North Carolina at Greensboro Greensboro, NC 27402, USA

E-mail address: shivaji@uncg.edu

TMNA : Volume $50-2017-\mathrm{N}^{\mathrm{O}} 1$ 